isw1g10@soton.ac.uk 


\title{
A novel approach to analysing fixed points in complex systems
}

\author{
Iain S. Weaver ${ }^{\star \star}$ and James G. Dyke \\ School of Electronics and Computer Science, University of Southampton
}

\begin{abstract}
Complex systems are frequently characterised as systems of many components whose interactions drive a plethora of emergent phenomena. Understanding the history and future behaviour of planet Earth, arguably the most complex known system in the universe, is an ambitious goal and remains at the core of complexity science. From the establishment of the planet's magnetic dipole, to the interplay between life and it's environment. The dynamics across all scales are characterised by their numerous interacting components.

Of particular interest is how such a system may be stable at all, and the role of life in establishing this apparent stability. We present a novel analytic approach to a model of a coupled life-environment system. The model demonstrates that even random couplings between many many species, and a multidimensional environment can produce stable, and robust configurations. The extent to which this observation is general, rather than being unique to the intricacies of the model may only be revealed by thorough analysis. The model is found to be invariant with the number of biotic components past a lower limit. Additionally, rather than increases in environmental complexity leading to a reduction in the possibility of steady states, it is proven that the converse is true, suggesting that the proposed mechanism may be applicable to even high dimensional complexity.
\end{abstract}

\section{Introduction}

The Gaia hypothesis proposes the idea that life on Earth and it's abiotic environment are tightly coupled in such a way as to maintain conditions to be within a range essential for life to exist (Lovelock 1979). This phenomena is commonly referred to as homeostasis; in the face of external and internal perturbations, factors such as surface temperature, and atmospheric composition appear to be tightly reigned. Indeed, catastrophic perturbations have occurred in Earth's past, characterised by mass extinction events and climatic shifts. In response, the system stabilises rather than being condemned to a lifeless state.

A number of mechanisms have been proposed for these phenomena (Lenton et al. 1998), perhaps most notably by the original proponent of the Gaia hypothesis himself. In the original "Daisyworld" model, Watson and Lovelock (1983)

\footnotetext{
** Corresponding author.

e-mail: isw1g10@soton.ac.uk
} 
seek to dispel the idea that the Gaia hypothesis relies on any sort of global control, rather that natural selection is sufficient. The model describes a grey planet, seeded by black and white daisies, orbiting a star of gradually increasing luminosity. Being abundant in all other factors required for the daisies to flourish, the model's principle variable is surface temperature. With the right choice of feedback the model exhibits homeostasis in that the temperature of the planet is maintained roughly constant in the face of an increasingly bright star, and life is maintained across a much greater range than might naïvly be predicted. While an important proof of concept for the Gaia hypothesis, spurring a great deal of additional research (see Wood et al. (2008) for an review of the developments in this area), it is difficult to see the Daisyworld mechanism as being generally applicable; the Earth system is characterised by numerous principle variables, and rather than a pair of competing species, a multitude of forms of life exist across a vast range of conditions. Furthermore, there is no reason to believe $a$ priori that life should organise into homeostatic states, as opposed to run-away positive feedback (Kirchner 1989).

The reorganisation of the Earth system in response to destabilising perturbations can be seen as an example of Ashby's (1952) notion of ultrastability. Ashby's "Homeostat" model was, in contrast to Daisyworld, a physical device which was able to respond to this type of perturbation with spontaneous reorganisation, through a random search mechanism. The result was that the systems variables are constrained within some limited range; a potential likeness with the Earth system.

Dyke (2010) proposes a model in which a large number of biotic components interact through a shared environment. The model is able to reproduce the salient features of both Ashby's (1952) Homeostat, and Watson and Lovelock's (1983) original Daisyworld model, without the need to prescribe a tendency for positive or negative feedback. This model of many biotic elements, interacting through their multidimensional environment serves as the starting point of this paper. In Sec. 2, we present the model in a simplified state, along with some characteristic results in Sec. 3. We then pose a number of potential criticisms which would conflict with the proposition of a general model in Sec. 4. We systematically address the model treatment of biotic complexity in Sec. 4.1, along with the environmental complexity in Sec. 4.2. These results are discussed along with the possibility of application to a broader class of complex system in Sec. 5 .

\section{Model formulation}

Throughout this article, we consistently used the boldface notation to identify vectors of many components, and subscript to identify individual elements,

$$
\boldsymbol{X}=\left[\begin{array}{c}
X_{1} \\
X_{2} \\
\vdots \\
X_{n}
\end{array}\right]
$$


Dyke's (2010) "Daisystat" model expresses life as $K$ biotic elements whose overall activity is influenced by the state of their shared environment, represented the $N$ variables in the vector $\boldsymbol{E}$. This environment is itself influenced by the biota; it's variables may be decreased or increased by the individual biotic elements, through consumption, excretion or some other process with no bias towards positive or negative feedback. In essence, the model consists of two principle assumptions:

i) Environment affects life Each biotic element in the system occupies a niche, the relatively narrow envelope of possible conditions in which it can respire, proliferate, or otherwise maintain activity. As we depart from the optima, the biotic element will tolerate the change to some extent, remaining active outside it's optimal conditions. Eventually we depart the niche of this biotic element, and it may die, become dormant, or otherwise inactive, while other biotic elements may be better suited to the new environment, and increase in activity. However, there are limits to this process. The environment must remain within some essential range for the biota to be active at all. While species may prefer a wide range of different ambient temperatures, it is hard to imagine life flourishing in ice, or steam.

We are primarily concerned with the fixed points of this model where the activity of each biotic element is given by it's steady state value. If the biota is able to reorganise sufficiently quickly compared to changes in the environment, the biotic elements can be said to exist at this environment-dependant steady-state activity, removing the need to explicitly incorporate time dependence. The steady-state activity, $\alpha(\boldsymbol{E})$, is maximised at a point in the space of environmental variables, $\boldsymbol{\mu}$, it's niche. As $\boldsymbol{E}$ departs from $\boldsymbol{\mu}$, the steady-state activity of this biotic element decreases. There are a range of defensible options for representing this behaviour, and we later investigate the extent to which this choice is important for the model dynamics. For simplicity, we choose the activity of an individual biotic element, $\alpha_{i}(\boldsymbol{E})$, to be a Gaussian, centred at $\boldsymbol{\mu}_{i}$ with characteristic width $\sigma_{E}$, that is

$$
\alpha_{i}(\boldsymbol{E})=\exp \left(-\frac{\left|\boldsymbol{E}-\boldsymbol{\mu}_{i}\right|^{2}}{2 \sigma_{E}^{2}}\right)
$$

where $\boldsymbol{\mu}$ is the optimum for an individual biotic element chosen randomly in the interval $[0: R]$, the essential range. For the purposes of this section, we use $\sigma_{E}=5, R=100$ and $K=10^{4}$ biotic elements, distributed across this range.

ii) Life affects environment The impact of life on the environment is hard to generalise. As stated, the Earth system is composed of numerous principle variables. Some of these variables are considered resources, consumed by some forms of life, and possibly excreted by others, such as oxygen and carbon dioxide in the atmosphere, or phosphorous in the soil. Other variables such as surface temperature are not consumed in this way, although species of foliage may have 
an effect on this variable indirectly by modifying the planetary albedo locally. Rather than make this distinction, we opt simply to impose random, and unbiased couplings between life, and it's environment.

The biotic elements may feedback negatively or positively, and strongly or weakly (or not at all), depending on the value of a weighting term. Whatever the weighting, we say their effect is directly proportional to their activity; an abundant, or highly active element is likely to have a more significant impact. The total biotic effect is found by summing the contributions of each element

$$
F_{i}(\boldsymbol{E})=\sum_{j=1}^{K} \omega_{j, i} \alpha_{j}(\boldsymbol{E})
$$

where $\omega_{j, i}$ is the weight of biotic element $j$ on environmental variable $i$, chosen randomly from the interval \pm 1 . Additionally, each of the environmental variables may be affected by some external perturbing force, $\boldsymbol{P}$. The net change in the environment is the sum of these features

$$
\tau_{E_{i}} \frac{\mathrm{d} E_{i}}{\mathrm{~d} t}=P_{i}+F_{i}
$$

where $\tau_{E_{i}}$ is the characteristic timescale for changes in environmental variable $i$, chosen to be equal between variables for convenience, and $F_{i}$ is the sum of effects from the biota.

Model behaviour Eq. (3) describes the time evolution of the environmental variables towards its fixed points, which occur where the sum of effects of the biotic elements on each resource exactly opposes the external perturbation on that resource, that is

$$
F_{i}=-P_{i} \quad \text { for } i=1 \ldots N .
$$

If they exist, these points may further be stable or unstable. If a change in $E_{i}$ results in an opposing change in $F_{i}$, the point is stable in this direction. A fixed point in the model must be stable in all directions, that is

$$
\frac{\mathrm{d} F_{i}}{\mathrm{~d} E_{i}}<0 \quad \text { for } i=1 \ldots N .
$$

\section{Model results}

Despite it's very general formulation, and largely random parameters, the model exhibits a range of interesting behaviour. The sum of uncorrelated Gaussians results in homeostatic behaviour in response to increasing perturbing force. This also leads to hysteresis loops illustrated for the case of a single environmental variable in Fig. 1. The behaviour is almost identical to Watson and Lovelocks Daisyworld model, except rather than homeostasis being designed, such points have emerged spontaneously from the biological complexity of the model. 

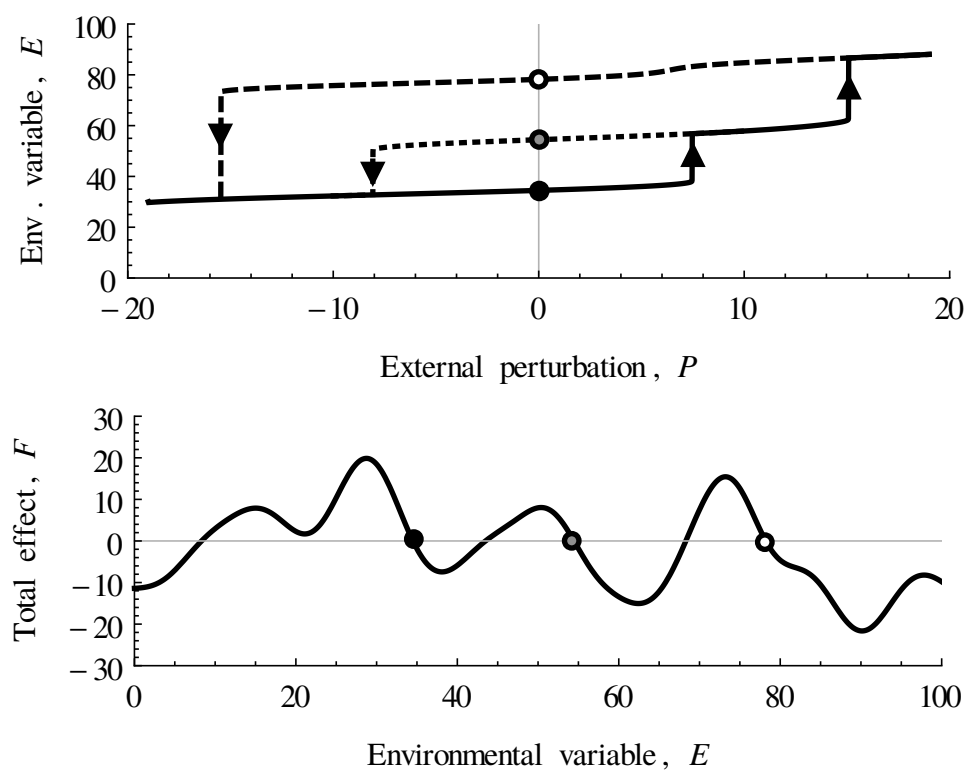

Fig. 1. The model has homeostatic fixed points, shown here for a single environmental variable. Population effects oppose increasing (solid line) or decreasing (dashed line) perturbations, maintaining a roughly constant environment (top). Hysteresis is caused by the existence of multiple solutions, shown for the case of $P=0$ (bottom). Sharp transitions into new stable states occur when such points vanish. 
A further criticism to the Daisyworld model is that it hinges on a single environmental variable, and therefore necessarily exhibits simple behaviour. On the other hand the Earth system could not possibly be reduced in this way (Kirchner 2003). Initial intuitions may suggest that with increasing environmental variables the likelihood of finding a point stable in all dimensions simultaneously would vanish exponentially. However, transitions between neighbouring attractive points can be seen in higher dimensional systems. A model of four environmental variables is shown to settle into a stable fixed point in Fig. 2, and further illustrates that stability can be re-established after a perturbation removes the system from the basin of attraction corresponding to this point, qualitatively similar to the four unit Homeostat. Fig. 3 directly examines the basins of attraction for a two dimensional model and illustrates the complicated structure of underlying basins of attraction.

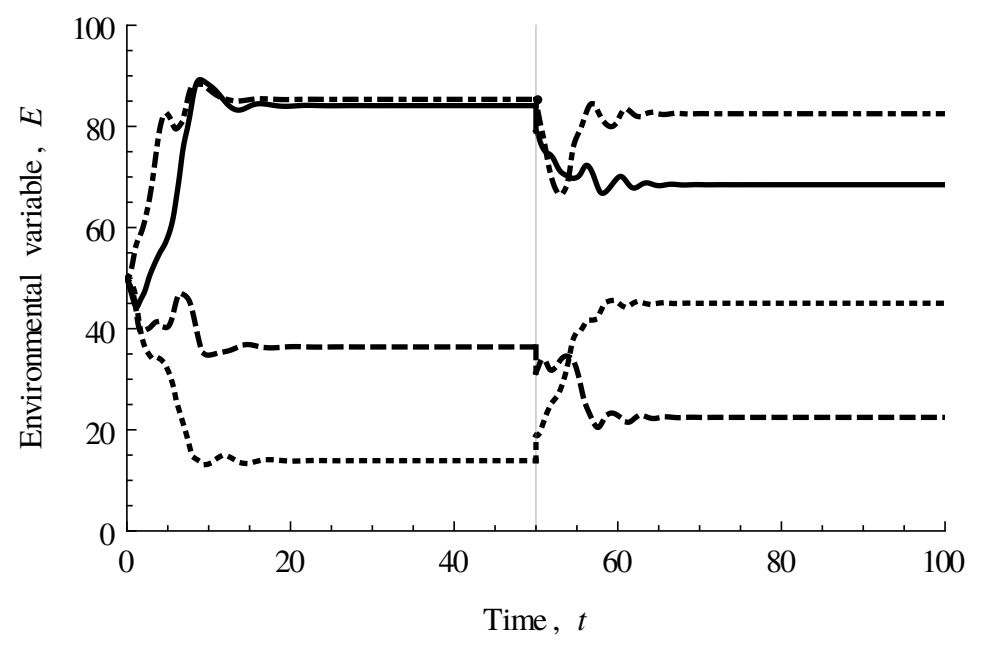

Fig. 2. The $N=4$ model, initialised with all environmental variables at the centre of the essential range. After reaching a steady-state, a perturbation is applied to the variables at time 50 which is sufficient for the model to enter a new attractor.

\section{Analysis}

While the model may give insights to key features of the Earth system, it is difficult to establish to what extent this may represent a general mechanism where homeostasis is inevitable, and if or how it is constrained by assumptions and implementation. Three of the key matters to address are; 


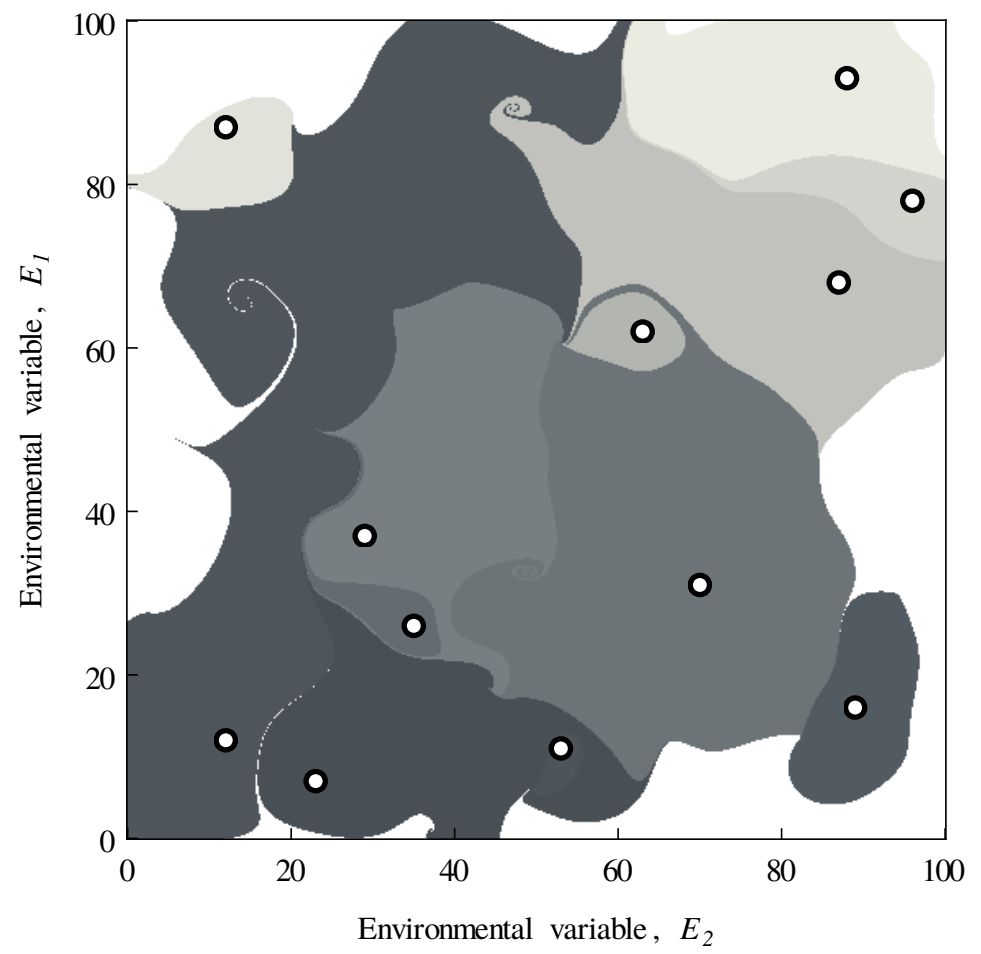

Fig. 3. The $N=2$ model shows many stationary points, indicated by points. The basins of attraction which lead to these points are indicated by the shaded enclosing regions, while initial conditions which would leave the essential range are coloured white. 
- Does the model behaviour change with increasing numbers of biotic components?

- How is it affected by increasing environmental complexity?

- To what extent is the choice of underlying functions important?

One may intuit that with very many biotic elements, there is a tendency towards uniformity in $\boldsymbol{F}$, reducing the likelihood of finding stationary points. While early intuitions were that increasingly complex systems enjoyed increased stability (Odum 1971), the work of Gardner and Ashby (1970) and May (1972) contended this, showing that in a network interpretation of complexity, increased numbers and strength of connections ultimately led to instability. In our model, fixed points must be stable in all environmental variables simultaneously, suggesting that with an increasingly complex environment stability becomes impossible.

\subsection{Behaviour with number of biotic elements, $K$}

We introduce the covariance function as a means to characterise the nature of the sum of biotic effects $\boldsymbol{F}$. The covariance function encodes the degree of correlation between points in $\boldsymbol{E}$. We write $k_{i}\left(\boldsymbol{E}, \boldsymbol{E}^{\prime}\right)$ as shorthand for the covariance of $F_{i}$, $\left\langle F_{i}(\boldsymbol{E}) F_{i}\left(\boldsymbol{E}^{\prime}\right)\right\rangle$, at two arbitrary points in the space of environmental variables, $\boldsymbol{E}$ and $\boldsymbol{E}^{\prime}$.

$$
k_{i}\left(\boldsymbol{E}, \boldsymbol{E}^{\prime}\right)=\left\langle\sum_{n, m=1}^{K} \omega_{i, n} \omega_{i, m} \alpha_{n}(\boldsymbol{E}) \alpha_{m}\left(\boldsymbol{E}^{\prime}\right)\right\rangle .
$$

At this point, we can exploit the absence of correlations first between individual biotic elements, and then between the weights $\omega$, and the biotic activity. The first observation leads us to conclude the off-diagonal terms, where $i \neq j$, do not contribute to the covariance. The second enables us to separate the expectation values of $\omega$ and $\alpha$, giving

$$
k_{i}\left(\boldsymbol{E}, \boldsymbol{E}^{\prime}\right)=K \sigma_{\omega}^{2}\left\langle\alpha(\boldsymbol{E}) \alpha\left(\boldsymbol{E}^{\prime}\right)\right\rangle .
$$

where $\sigma_{\omega}^{2}$ is the variance of the random variable $\omega$. The right side of this equation can be identified simply as the covariance of the individual biotic activity functions. This result illustrates that the covariance of the summed functions share the functional form of the individual functions of which it is comprised. The characteristic length, and therefore the propensity for $\boldsymbol{F}$ to form attractive fixed points, is independent of the biotic complexity of the model. This can be verified numerically by examining how the expected number of fixed points in a single variable model varies with the number of biotic elements, $K$. Fig. 4 illustrates that the number of fixed points saturates quickly, and that further increasing $K$ does not modify the behaviour of the model.

\subsection{Behaviour with number of environmental variables, $N$}

A fixed point in $F_{i}(\boldsymbol{E})$ occurs in a small interval of $\boldsymbol{E}$ if its sign changes across the interval. Labelling the interval $\boldsymbol{\epsilon}$, this condition can be expressed

$$
F_{i}(\boldsymbol{E}) F_{i}(\boldsymbol{E}+\boldsymbol{\epsilon})<0
$$




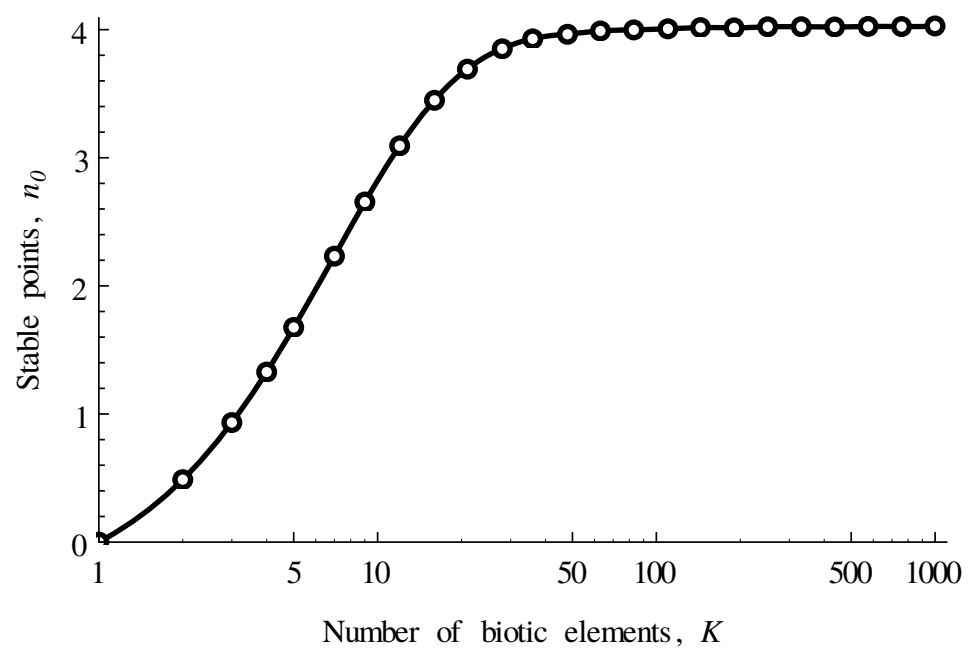

Fig. 4. The number of fixed points increases and then saturates with increasing number of biotic components $K$. The number of fixed points linearly decreases with increasing the width of the biotic component abundance function $\sigma_{E}$ and linearly increases with increases in the width of the essential range $R$

and the expected number of such points in the unit interval, $n_{0}$, is found from a product of indicator functions of the form of Eq. (8)

$$
n_{0}=\left\langle\prod_{i=1}^{N} \frac{1}{\epsilon}\left[F_{i}(\boldsymbol{E}) F_{i}(\boldsymbol{E}+\boldsymbol{\epsilon})<0\right]\right\rangle .
$$

We have used [...] to represent an indicator function, returning one if the expression true, and zero otherwise. The expectation of an indicator function may be interpreted as the probability of it's contents being true, and the product of several therefore gives the probability of many conditions being met simultaneously. Each term in the product may be treated independently due to the independence between the biotic effects on the different environmental variables $F_{i}$ and $F_{j}$. The problem is therefore reduced to finding the value of the series of $N$ expectation values. Expanding for small $\epsilon$ gives

$$
p=\left\langle\left[F_{i}(\boldsymbol{E})<-\epsilon F_{i}^{\prime}(\boldsymbol{E})\right]\right\rangle
$$

where $F_{i}^{\prime}(\boldsymbol{E})$ is used to indicate the derivative of $F_{i}(\boldsymbol{E})$ in the $\hat{\boldsymbol{\epsilon}}$ direction (commonly written as $\nabla_{\hat{\epsilon}} F_{i}(\boldsymbol{E})$ ). To find the expectation value of this indicator function, we need to know how $F_{i}(\boldsymbol{E})$ and $F_{i}^{\prime}(\boldsymbol{E})$ are distributed. Rather than suffer any loss of generality, we make three important observations. Firstly, at any point in $\boldsymbol{E}$ within the essential range, $F_{i}(\boldsymbol{E})$ is a sum of independent contributions from the biotic elements. Therefore, by the central limit theorem, each point follows a Gaussian distribution. Additionally, this distribution has a mean 
of zero as previously stated. There is no tendency for positive or negative feedback between the biota and environment. Finally, we note $F_{i}(\boldsymbol{E})$ and $F_{i}^{\prime}(\boldsymbol{E})$ to be uncorrelated as a consequence of our independent parameters $\boldsymbol{\mu}$ and $\omega$.

The problem is now dramatically reduced, we need only find the variance of the Gaussian random variables $F_{i}(\boldsymbol{E})$ and $F_{i}^{\prime}(\boldsymbol{E})$, labelled $\sigma_{F}^{2}$ and $\sigma_{F^{\prime}}^{2}$ respectively. Here it is useful to observe that providing the width of the biotic activity functions are small compared to the essential range, the covariance $k\left(\boldsymbol{E}, \boldsymbol{E}^{\prime}\right)$ is stationary; $k$ depends only on the distance $\left|\boldsymbol{E}-\boldsymbol{E}^{\prime}\right|$. Having already determined the covariance of $F_{i}(\boldsymbol{E})$ in Eq. (7), the variance therefore may be written as

$$
\sigma_{F}^{2}=k_{i}(\mathbf{0}) .
$$

We can write a similar expression for $F_{i}(\boldsymbol{E})$, and remove the directional derivative from the expectation value to give

$$
\begin{aligned}
\sigma_{F^{\prime}}^{2} & =\left.\left\langle F^{\prime}(\boldsymbol{E}) F^{\prime}\left(\boldsymbol{E}^{\prime}\right)\right\rangle\right|_{\boldsymbol{E}=\boldsymbol{E}^{\prime}} \\
& =\left.\nabla_{\boldsymbol{E}, \hat{\boldsymbol{\epsilon}}} \nabla_{\boldsymbol{E}^{\prime}, \hat{\boldsymbol{\epsilon}}} k_{i}\left(\boldsymbol{E}-\boldsymbol{E}^{\prime}\right)\right|_{\boldsymbol{E}=\boldsymbol{E}^{\prime}} \\
& =-k_{i}^{\prime \prime}(\mathbf{0}) .
\end{aligned}
$$

Next, we substitute Eqs. (11) and (12) into Eq. (10)

$$
p=\iint\left[F_{i}<-\epsilon F_{i}^{\prime}\right] P\left(F_{i}\right) P\left(F_{i}^{\prime}\right) \mathrm{d} F_{i} \mathrm{~d} F_{i}^{\prime}
$$

where $P\left(F_{i}\right)$ and $P\left(F_{i}^{\prime}\right)$ are the Gaussian distributions

$$
\begin{aligned}
& P\left(F_{i}\right)=\frac{1}{\sqrt{2 \pi} \sigma_{F}} \exp \left(-\frac{F_{i}^{2}}{2 \sigma_{F}^{2}}\right), \\
& P\left(F_{i}^{\prime}\right)=\frac{1}{\sqrt{2 \pi} \sigma_{F^{\prime}}} \exp \left(-\frac{F_{i}^{\prime 2}}{2 \sigma_{F^{\prime}}^{2}}\right) .
\end{aligned}
$$

After a change of variable, $\frac{F_{i}}{\sigma_{F}} \rightarrow x$ and $\frac{F_{i}^{\prime}}{\sigma_{F^{\prime}}} \rightarrow x^{\prime}$, we can exploit spherical symmetry in $x$ and $x^{\prime}$ to find the expectation of the indicator function to be

$$
\begin{aligned}
p & =\iint\left[x \sigma_{F}<-\epsilon x^{\prime} \sigma_{F^{\prime}}\right] \frac{e^{-\frac{x^{2}}{2}}}{\sqrt{2 \pi}} \frac{e^{-\frac{x^{\prime 2}}{2}}}{\sqrt{2 \pi}} \mathrm{d} x \mathrm{~d} x^{\prime} \\
& =\frac{1}{\pi} \operatorname{atan}\left(\epsilon \frac{\sigma_{F^{\prime}}}{\sigma_{F}}\right)
\end{aligned}
$$

which can be expanded to first order for small $\epsilon$, and substituted into Eq. (9) to give

$$
n_{0}=\left(\frac{p}{\epsilon}\right)^{N}=\left(\frac{1}{\pi} \sqrt{-\frac{k^{\prime \prime}(\mathbf{0})}{k(\mathbf{0})}}\right)^{N}
$$

which is consistent with Alder and Strassen (1981) for the case of a one-dimensional model (Theorem 4.1.1). Counter to intuition, the number of stable fixed points 
within the essential range of the model may increase exponentially, rather than vanishing to zero providing there is a sufficiently wide essential range. For the simple example of Gaussian functions the expected number of fixed points is

$$
n=\left(\frac{R}{\sqrt{2} \pi \sigma_{E}}\right)^{N},
$$

of which the fraction $2^{-N}$ are attractive. Eq. (18) makes clear the role of the width of biotic activity functions in guiding the model, while the specific function chosen is unimportant. Indeed, skewed, bimodal and to some extent, long-tailed functions can be shown to produce similar behaviour. Not only may very high dimensional systems exist in stable, stationary states, but the number of such states may be exponentially great. As before, we can verify this relationship numerically up to four environmental variables, shown in Fig. 5.

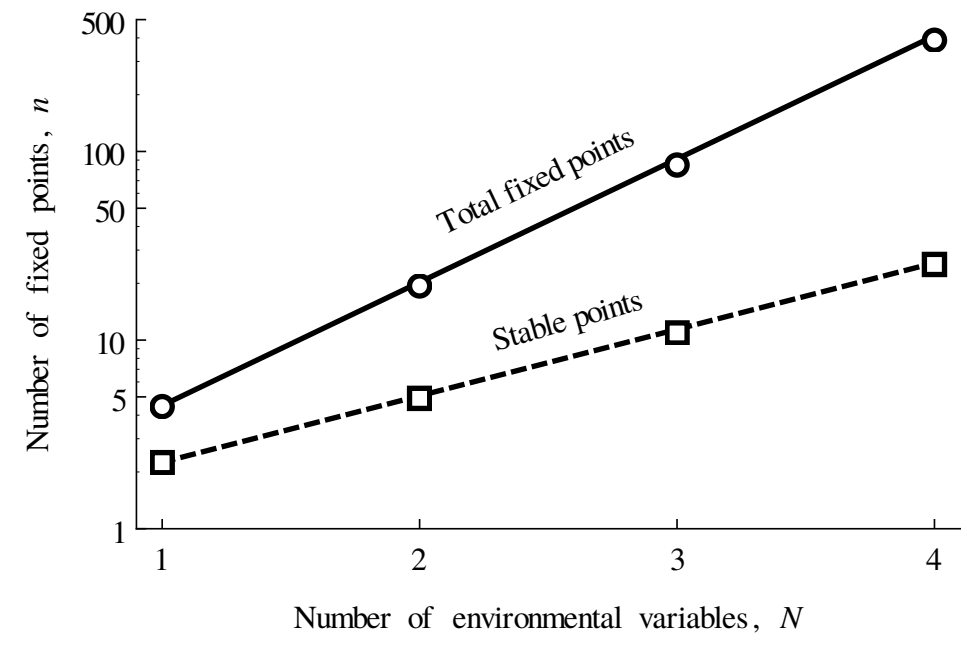

Fig. 5. Eq. (17) can be used to find the expected number of fixed points across the essential range with an increasingly complex environment. Numerical simulations are plotted as points, where statistical errors are at most the size of plot points. Simulation confirms the exponential increase in fixed points with the addition of environmental variables.

\section{Conclusion}

It has been demonstrated that collections of biotic elements interacting with a complex environment in randomly parametrised ways can not only reach stable configurations which are robust to external perturbations, but display a plethora 
of complex phenomena. The extent to which this is a generally applicable principle is addressed by an analytic investigation into the model behaviour with increasing biotic, and environmental complexity.

By observing that the sum of many functions shares the covariance function of it's components, we find that rather than a tendency towards uniformity, increasing the number of biotic elements does not hinder the ability of the model to form attractive fixed points. A relatively sparse biota display almost identical properties to one containing many times more elements.

Furthermore, we exploit the Gaussian nature of sums of random biotic effects to derive expressions for the expected number of model fixed points for given dimensionality. In doing so, we find the choice of biotic activity function to be largely arbitrary. Rather than multidimensional systems lacking attractive fixed points, we find the number of such points can increase exponentially with the dimensionality, at a rate determined by the characteristic width of the individual biotic activity functions.

Through this analysis, we have preserved generality where possible, though a number of significant assumptions are made. The covariance of the total biotic effects is approximated by a stationary function, a condition that requires the width of individual activity functions to be small compared to the essential range for life. Additionally it is assumed that the number of biotic elements is sufficiently large that our reliance on the central limit theorem is justified. While numerical validation is provided here, the extent to which these conditions are met by real systems may be the main limitation of this approach.

\section{Acknowledgements}

This work was supported by an EPSRC Doctoral Training Centre grant (EP/G03690X/1). 


\section{Bibliography}

Alder, A., Strassen, V.: On the algorithmic complexity of associative algebras. Theoretical Computer Science 15(2), 201-211 (1981)

Ashby, W.: Design for a brain. Wiley (1952)

Dyke, J.G.: The daisystat: A model to explore multidimensional homeostasis. In: Artificial Life XI, Proceedings of the Eleventh International Conference on the Simulation and Synthesis of Living Systems. pp. 349-359. MIT Press, Cambridge MA (2010)

Gardner, M., Ashby, W.: Connectance of large dynamic (cybernetic) systems: critical values for stability. Nature 228, 784 (1970)

Kirchner, J.: The gaia hypothesis: can it be tested. Rev. Geophys 27(2), 230 (1989)

Kirchner, J.: The gaia hypothesis: conjectures and refutations. Climatic Change 58(1), 21-45 (2003)

Lenton, T., et al.: Gaia and natural selection. Nature 394(6692), 439-447 (1998)

Lovelock, J.: A new look at life on earth. Ox-ford University Press, Oxford (1979)

May, R.: Will a large complex system be stable? Nature 238, 413-414 (1972)

Odum, E.: Fundamentals of ecology. WB Saunders Co (1971)

Watson, A., Lovelock, J.: Biological homeostasis of the global environment: the parable of daisyworld. Tellus B 35(4), 284-289 (1983)

Wood, A.J., Ackland, G.J., Dyke, J.G., Williams, H.T.P., Lenton, T.M.: Daisyworld: a review. Reviews of Geophysics 46, RG1001 (2008) 